\title{
PROPAGATION AND REFLECTION OF MAGNETO-ELASTIC PLANE WAVES AT THE FREE SURFACE OF A ROTATING MICROPOLAR FIBRE-REINFORCED MEDIUM WITH VOIDS
}

\author{
Augustine Igwebuike Anya, Aftab Khan \\ COMSATS University Islamabad, Department of Mathematics, Islamabad, Pakistan \\ e-mail: anyaaugustineigwebuike@gmail.com
}

\begin{abstract}
This work investigates rotational effects on propagation and reflection of waves at the free surface of a micropolar fibre-reinforced medium with voids under magnetic fields. When the $\mathrm{P}$-wave is incident on the free surface, there exist four coupled reflected plane waves traveling in the medium; quasi-longitudinal displacement (qLD) wave, quasi-transverse displacement (qTD) wave, quasi-transverse microrotational (qTM) wave and a wave due to voids. Normal mode analysis is adopted in concomitant with Snell's laws, and appropriate boundary conditions in determination of the solution. Amplitude ratios which correspond to reflected waves in vertical and horizontal components are presented analytically and graphically.

Keywords: rotation, micropolar fibre-reinforced, voids, reflection coefficients, magnetic field
\end{abstract}

\section{Introduction}

Fibre-reinforced composite materials and their analysis of stress and deformation are of great interest to researchers in solid mechanics. This is as a result of its weightlessness and high tensile strength. Structural designers mostly in the field of engineering, science and technology, usually sought after such type of materials. However, many researchers have investigated wave propagation in various materials or media. But a particular investigation of isotropic generalized solids with voids paved way as an evidence to the existence of new or transformed waves, whose importance to seismologists in improving earthquake estimations cannot be over emphasized. In geophysics, for instance, the reflection and refraction of seismic waves gave credence to researchers to investigate the earth's interior and structures. Thus, it is obvious that most large bodies such as planets, moon and earth possess angular velocity. Consequently, this necessitates the study of rotational effects on reflection of plane waves. Moreover, the frame of reference of rotation of the medium, centrifugal acceleration and Coriolis effects are taken into considerations in the equation of motion.

On the other hand, micro-continuum as a material property due to deformation consists of microstretch, micropolar and micromorphic theories, and this depends on the order to which the micro-degree is incorporated. Eringen (1967) developed theory from micropolar elasticity to micropolar linear constitutive theories with internal friction. McCarthy and Eringen (1969) investigated the problem on micropolar viscoelastic waves. Kumar et al. (1990) studied problems associated with Lamb's plane micropolar viscoelastic medium with stretch. Biswas et al. (1996) studied the axisymmetric Lamb's problem of a micropolar viscoelastic medium. Singh and Kumar (1998) investigated the amplitude ratios of reflection and refraction of plane waves between a micropolar elastic solid and viscoelastic solid interfaces. Singh (2000) developed the reflection/transmission between a liquid and micro-polar viscoelastic solid interfaces with stretch. Nunziato and Cowin (1979) and Cowin and Nunziato (1983) discussed both linear and nonlinear 
theories of elastic porous media. Puri and Cowin (1985) studied the effects of voids on plane waves in linear elastic media. Singh (2017) gave account of reflection of elastic plane surface waves of a half-space with impedance boundary conditions. Sengupta and Nath (2001) discussed surface waves in fibre-reinforced media. Chattopadhyay et al. (2002) studied reflection of quasi-SV and quasi-P waves using both free and rigid boundary conditions of a fibre-reinforced medium. Chaudhary et al. (2004) envisaged reflection/transmission of plane waves between self-reinforced two elastic half-spaces. Khan et al. (2015) studied the effects of surface waves under the influence of gravity in a non-homogeneous fibre-reforced media with voids. Tauchert (1971) investigated thermal stresses in micropolar elastic solids. Chattopadhyay and Choudhury (1990) obtained results on propagation, reflection/transmission of waves under the magnetic effects in a self-reinforced medium. Kumar et al. (2015) worked on reflection of plane waves in transversely isotropic micropolar viscoelastic media with thermal effects. Furthermore, Abd-Alla et al. (2017) investigated rotational behavior of magneto-thermoelastic surface waves in fibre-reinforced viscoelastic media of higher order. Lata (2018) studied reflection and refraction of plane waves in a layered nonlocal elastic and anisotropic thermoelastic medium. Schoenberg and Censor (1973) examined the propagation of waves in a rotating, homogeneous, isotropic, linear elastic medium. An eigenvalue approach was utilized by Sinha and Bera (2003) to solve the fundamental equations of problems of generalized thermoelasticity with one relaxation parameter including the heat source in an infinite rotating medium. Sunita et al. (2019) examined reflection on a free surface of a fibre-reinforced thermoelastic rotating medium with two-temperature and phase-lag. Roy et al. (2017), examined propagation and reflection of plane waves in a rotating magnetoelastic fibre-reinforced semi space with surface stress. Hillion (2008) investigated harmonic plane wave propagation in anisotropic chiral media.

In the light of the above literature, the current study envisages propagation and reflection of magneto-elastic plane waves at free surfaces of a rotating micropolar fibre-reinforced medium with porosity under magnetic fields. That is, interactions between the magnetic field and the rotating micropolar fibre-reinforced medium with voids or porosity in the $x_{1} x_{2}$-plane is considered. Due to micropolarity, the coefficient matrices in the constituent relations are non-symmetric i.e., the stress tensors and deformation tensors are considered to be non-symmetric. Thus, the micropolar fibre-reinforced problem in $2-\mathrm{D}$ is modelled. The governing equations are solved by using free boundary conditions also called natural boundary conditions. We observed that four reflected waves exist when, in particular, the $\mathrm{P}$-wave is incident on the free surface or at the boundary $x_{2}=0$; quasi-longitudinal displacement (qLD) or P-wave, quasi-transverse displacement (qTD) or SV-wave, quasi-transverse microrotational (qTM) wave and a wave due to voids. Amplitude ratios or reflection coefficients which correspond to the reflected waves in both horizontal and vertical components are presented analytically. Also, by using Mathematica software, computational results which stipulate the effects of rotation, magnetic field, fibre-reinforced and voids parameters for the horizontal reflection coefficients are shown graphically. Some particular results can also be deduced in the absence of rotation, magnetic field and fibre-reinforced parameters, yielding the results of a micropolar isotropic solid with voids.

\section{Formulation of the problem}

The basic field relations for a micro-polar fibre-reinforced homogenous linearly elastic anisotropic medium with the reinforcement direction a and voids (Eringen, 1967; Kumar et al., 2015; Lakes and Benedict, 1982; Cowin and Nunziato, 1983) are specified by

$$
\sigma_{i j}=B_{i j m n} E_{m n}+N_{i j m n} \psi_{m n}+\xi \phi \delta_{i j} \quad m_{i j}=B_{j i m n} E_{m n}+N_{m n j i} \psi_{m n}
$$

The deformations and wryness tensors are taken as

$$
E_{i j}=u_{j, i}+\varepsilon_{j i m} \phi_{m}^{*} \quad \psi_{m n}=\phi_{m, n}^{*} \quad i=j=m=n=1,2,3
$$


The balance laws in the presence of rotation, magnetic fields and voids (Abd-Alla et al., 2017; Eringen, 1967; Cowin and Nunziato, 1983) are written below

$$
\begin{aligned}
& \sigma_{i j, i}+F_{j}=\rho\left(\ddot{u}_{j}+\Omega_{i} u_{i} \Omega_{j}-\Omega^{2} u_{j}+2 \varepsilon_{j i k} \Omega_{i} \dot{u}_{k}\right) \\
& m_{i j, i}+\varepsilon_{j m n} \sigma_{m n}=\rho J \ddot{\phi}_{j}^{*} \\
& \xi_{1}\left(\phi_{, i i}\right)-\omega_{0} \phi-\varpi \dot{\phi}-\xi\left(u_{i, i}\right)=\rho \kappa \ddot{\phi}
\end{aligned}
$$

Let the solid medium be rotating about the $x_{3}$-axis with uniform angular velocity $\Omega$, i.e. $\boldsymbol{\Omega}=\Omega(0,0,1)$, where $\sigma_{i j}\left[\mathrm{~N} / \mathrm{m}^{2}\right], m_{i j}\left[\mathrm{~N} / \mathrm{m}^{2}\right], \phi_{j}^{*}, u_{j}[\mathrm{~m}]$ and $\phi$ are the stress tensor, couple stresses, microrotation vector, displacement vector and volume fraction field, respectively. Also, $\rho, \xi$ and $J\left[\mathrm{~m}^{2}\right]$ represent the bulk mass density, void parameter and micronertia respectively. $B_{i j m n}, B_{j i m n}$ and $N_{i j m n}$ are characteristic constants of the material, and also non symmetric properties of $B_{i j m n}, B_{j i m n}$ and $N_{i j m n}$ hold. For simplicity, we chose $\mathbf{a}=\left[a_{1}, a_{2}, a_{3}\right]$ such that $\mathbf{a}=[1,0,0]$ as the fibre direction. $\delta_{i j}$ is the Kronecker-delta function, $\varepsilon_{j i m}$ is the Levi-Civita alternating symbol or tensor. The index after comma represents partial derivative with respect to coordinate and the superscript dot specifies partial derivative with respect to time. Consider deformation in the $x_{1} x_{2}$-plane and the microrotation vector as $\phi^{*}=\left[0,0, \phi_{3}^{*}\right]$. Also Einstein summation convention over repeated indexes is used. However, $F_{i}=\mu_{0} \varepsilon_{i j k} J_{j} H_{k}$ and we take the linearized Maxwell equations governing the electromagnetic field for a perfectly conducting medium as

$$
\begin{array}{ccc}
\varepsilon_{i j k} H_{k, j}=\varepsilon_{0} \varepsilon_{i j k} J_{j} \dot{E}_{k} \quad \varepsilon_{i j k} E_{k, j}=-\mu_{0} \dot{H}_{i} & H_{i, i}=0 \\
E_{i, i}=0 & E_{i}=\mu_{0} \varepsilon_{i j k} \dot{u}_{j} H_{k} &
\end{array}
$$

where $H_{i}=H_{0} \delta_{i 3}+h_{i}, h_{i}$ is the induced magnetic field and $\varepsilon_{0}$ is electric permeability in farads $/$ meters $[\mathrm{F} / \mathrm{m}]$, and the material lies in the $x_{1} x_{2}$-plane. Thus, $H_{i}=H_{0} \delta_{i 3}+h_{i}$. Then the magnetic force is given as follows: $F_{i}=\mu_{0} H_{0}^{2}\left(e_{, i}-\varepsilon_{0} \mu_{0} \ddot{u}_{i}\right), F_{3}=0$ and $h_{i}\left(x_{1}, x_{2}, x_{3}\right)=-u_{k, k} \delta_{i 3}$, where $e=u_{1,1}+u_{2,2}$. In these equations, $F_{i}$ represents the magnetic force in newtons [N], $J_{i}$ is the current density in $\mathrm{A} / \mathrm{m}^{2}, H_{i}$ is the magnetic field vector having SI unit as ampere per meter $[\mathrm{A} / \mathrm{m}]$, and $\mu_{0}$ is magnetic permeability, measured in henry per metre $[\mathrm{H} / \mathrm{m}]$. Considering the fact that the tensors are non-symmetric, Eqs. (2.3) in the component form can be written as follows

$$
\begin{aligned}
& B_{1} u_{1,11}+\left(B_{2}+B_{3}\right) u_{2,12}+B_{4} u_{1,22}+B_{1}^{*} \phi_{3,2}^{*}+\xi \phi_{, 1}=\varepsilon_{0} \mu_{0}^{2} H_{0}^{2} \ddot{u}_{1}+\rho\left(\ddot{u}_{1}-\Omega^{2} u_{1}-2 \dot{u}_{2} \Omega\right) \\
& B_{5} u_{2,11}+B_{2} u_{1,12}+B_{6} u_{2,22}-B_{5} \phi_{3,1}^{*}+\xi \phi_{, 2}=\varepsilon_{0} \mu_{0}^{2} H_{0}^{2} \ddot{u}_{2}+\rho\left(\ddot{u}_{2}-\Omega^{2} u_{2}+2 \dot{u}_{1} \Omega\right) \\
& B_{5} \phi_{3,11}^{*}+B_{4} \phi_{3,22}^{*}-2 B_{4} \phi_{3}^{*}+B_{4}\left(u_{2,1}-u_{1,2}\right)=\rho J \ddot{\phi}_{3}^{*} \\
& \xi_{1}\left(\phi_{, 11}+\phi_{, 22}\right)-\omega_{0} \phi-\varpi \dot{\phi}-\xi\left(u_{1,1}+u_{2,2}\right)=\rho \kappa \ddot{\phi}
\end{aligned}
$$

where

$$
\begin{array}{lcr}
\left.B_{1}=\lambda+\beta+2 \alpha-2 \mu_{T}+4 \mu_{L}+\mu_{0} H_{0}^{2}\right) & B_{2}=\lambda+\alpha+\mu_{0} H_{0}^{2} \\
B_{3}=2\left(\mu_{L}-\mu_{T}\right) & B_{4}=2 \mu_{T} \quad B_{5}=2 \mu_{L} \\
B_{6}=\lambda+2 \mu_{T}+\mu_{0} H_{0}^{2} & B_{1}^{*}=B_{4}-B_{3}
\end{array}
$$

Also $\lambda$ represents Lame's material constant, $\alpha, \beta$ and $\left(\mu_{L}-\mu_{T}\right)$ are the fibre-reinforced parameters. All these parameters define the anisotropic model. If we consider $\mu_{L}=\mu_{T}=\mu$ (Lame's constant) and $\alpha=\beta=0$, the simple isotropic case is achieved.

\section{Normal modes analysis and solution of the problem}

Consider a rotating homogeneous micropalar fibre-reinforced anisotropic magneto-elastic medium with voids occupying the half-space $x_{2} \leqslant 0$ under the influence of a magnetic field. And 
let the plane waves be incident at the free boundary $x_{2}=0$ at an angle $\theta$ with the $x_{2}$-axis. Let the normal mode analysis be applicable such that the incident waves have the following displacements chosen as

$$
\left\{u_{j}, \phi^{*}, \phi\right\}=\left\{R, P, \phi_{0}^{*}, \phi_{0}\right\} \mathrm{e}^{\mathrm{i}\left[k\left(x_{1} \sin \theta+x_{2} \cos \theta\right)-\omega t\right]} \quad j=1,2
$$

where $R, P, \phi_{0}^{*}$ and $\phi_{0}$ are amplitudes of $u_{1}, u_{2}, \phi_{3}^{*}$ and $\phi$, measured in meters, respectively. $\omega$ is the angular velocity in radians per second, $c=\omega / k$ is the phase velocity of the wave in meter per second, and $k$ is the wave number and $(\sin \theta, \cos \theta)$ represents the projection of waves normal onto the $x_{1} x_{2}$-plane. We make use of Eq. (3.1) into Eqs. (2.4), respectively, which yields

$$
\begin{aligned}
& \left\{D_{1}-\left[\frac{\Omega^{2}}{k^{2}} \rho+c^{2}\left(\varepsilon_{0} \mu_{0}^{2} H_{0}^{2}+\rho\right)\right]\right\} R+\left[\left(B_{2}+B_{3}\right) \cos \theta \sin \theta+2 \mathrm{i} c \frac{\Omega}{k} \rho\right] \\
& \quad-\mathrm{i}\left(B_{1}^{*} \frac{1}{k} \cos \theta\right) \phi_{0}^{*}-\mathrm{i}\left(\xi \frac{1}{k} \sin \theta\right) \phi_{0}=0 \\
& \left(B_{2} \cos \theta \sin \theta-2 \mathrm{i} c \frac{\Omega}{k} \rho\right) R+\left\{D_{2}-\left[\frac{\Omega^{2}}{k^{2}} \rho+c^{2}\left(\varepsilon_{0} \mu_{0}^{2} H_{0}^{2}+\rho\right)\right]\right\} P \\
& \quad+\mathrm{i}\left(B_{5} \frac{1}{k} \sin \theta\right) \phi_{0}^{*}-\mathrm{i}\left(\xi \frac{1}{k} \cos \theta\right) \phi_{0}=0 \\
& \left(\mathrm{i} k B_{4} \cos \theta\right) R-\left(\mathrm{i} k B_{4} \sin \theta\right) P+\left(k^{2} D_{3}+2 B_{4}-J k^{2} \rho c^{2}\right) \phi_{0}^{*}=0 \\
& (\mathrm{i} \xi k \sin \theta) R+(\mathrm{i} \xi k \cos \theta) P+\left(\xi_{1} k^{2}+\omega_{0}-\varpi \mathrm{i} k c-\rho \kappa k^{2} c^{2}\right) \phi_{0}=0
\end{aligned}
$$

where $D_{1}=B_{1} \sin ^{2} \theta+B_{4} \cos ^{2} \theta, D_{2}=B_{5} \sin ^{2} \theta+B_{6} \cos ^{2} \theta$, and $D_{3}=B_{5} \sin ^{2} \theta+B_{4} \cos ^{2} \theta$. For a non-trivial solution, Eqs. (3.2) becomes the quartic equation as follows

$$
r^{4}+H_{1} r^{3}+H_{2} r^{2}+H_{3} r+H_{4}=0
$$

where $r=c^{2}$ which means that characteristic Eq. (3.3) with complex material coefficients $H_{1}, H_{2}, H_{3}$ and $H_{4}$, yields four distinct complex roots; detailing that four waves propagate, with complex phase velocities $c_{1}, c_{2}, c_{3}$ and $c_{4}$ corresponding to the wave numbers $k_{1}, k_{2}, k_{3}$ and $k_{4}$, respectively. So this means that the two dimensional model of reflection and propagation of magneto-elastic plane waves in a rotating micropolar fibre-reinforced half space medium with voids under consideration have four reflected waves; quasi-P wave, quasi-SV wave, quasitransverse microrotational wave and a wave due to voids travelling in the medium, if we assume that anyone of the four waves is incident at the free surface of the material.

\section{Reflection of plane waves at the free surface}

We consider the case when the quasi-P wave $\left(A_{0}\right)$ is incident at the boundary $x_{2}=0$ of the rotating micropolar fibre-reinforced anisotropic semi-infinite medium with voids under the influence of magnetic fields. Then, there exists four coupled reflected waves as the quasi-P $\left(A_{1}\right)$, quasi-SV $\left(A_{2}\right)$, quasi-TM $\left(A_{3}\right)$ and due to void $\left(A_{4}\right)$, see Fig. 1.

Thus, the total displacements can be assumed in the following form

$$
\left\{u_{1}, u_{2}, \phi_{3}^{*}, \phi\right\}=\sum_{l=0}^{4}\left\{R_{l}, P_{l}, \phi_{0}^{*}, \phi_{0}\right\} \mathrm{e}^{\mathrm{i} d_{l}}
$$

where

$$
\begin{array}{ll}
d_{0}=k_{0}\left[x_{1} \sin (e)+x_{2} \cos (e)-c_{1} t\right] & d_{1}=k_{1}\left[x_{1} \sin (e)-x_{2} \cos (e)-c_{1} t\right] \\
d_{2}=k_{2}\left[x_{1} \sin (f)-x_{2} \cos (f)-c_{2} t\right] & d_{3}=k_{3}\left[x_{1} \sin (g)-x_{2} \cos (g)-c_{3} t\right] \\
d_{4}=k_{4}\left[x_{1} \sin (h)-x_{2} \cos (h)-c_{4} t\right] &
\end{array}
$$




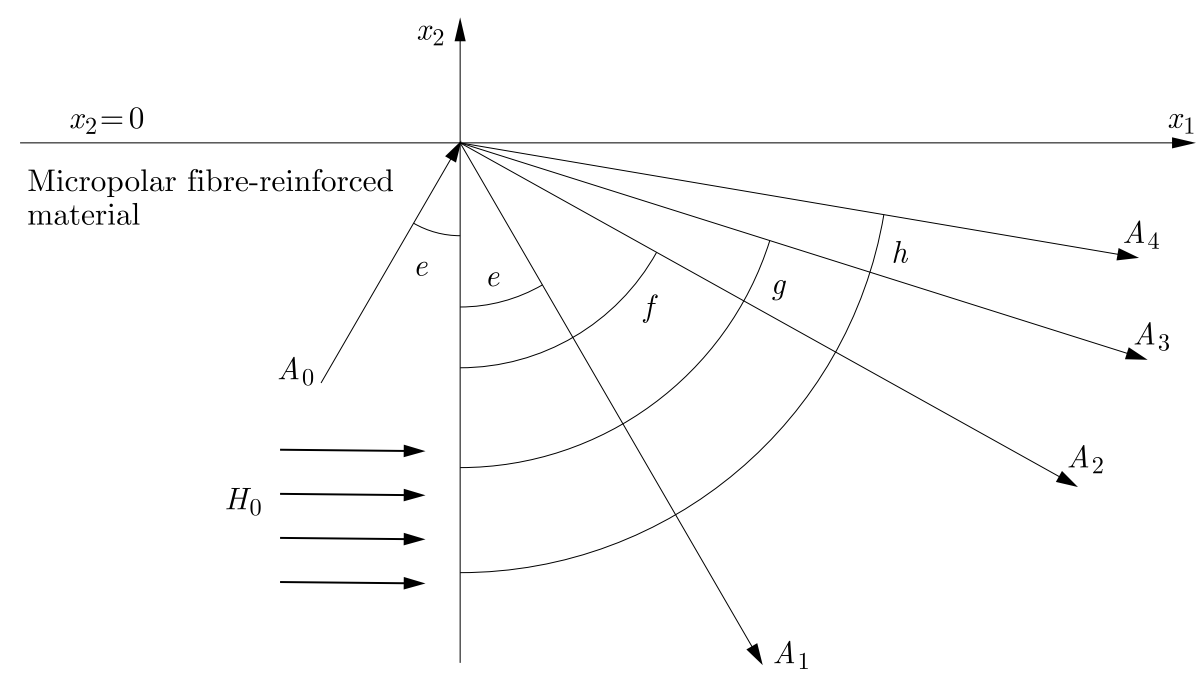

Fig. 1. Schematic of the problem showing incident and reflected waves

and $R_{0}$ is the amplitude of the incident wave while $R_{l}, l=1,2,3,4$, are amplitudes of the reflected waves in the horizontal components. Similarly, $P_{0}$ is the amplitude of the incident wave in the vertical components while $P_{l}, l=1,2,3,4$ are amplitudes of reflected waves in the vertical components. Incident and reflected waves given by (4.1) must satisfy equations of motion $(2.4)_{1,2}$ as a result of the consistency condition; following (Roy et al., 2017; Baljeet and Sarva, 2004), Eq. $(2.4)_{1}$ becomes

$$
\begin{aligned}
& {\left[D_{1}(e)-l_{1}\right] R_{0}+\left[\left(B_{2}+B_{3}\right) \sin (e) \cos (e)+2 \mathrm{i} c_{1} \frac{\Omega}{k_{1}} \rho\right] P_{0}} \\
& -\mathrm{i}\left[\frac{\mathrm{i}}{k_{0}} B_{1}^{*} \cos (e)\right] \phi_{0}^{*}-\left[\frac{\mathrm{i}}{k_{0}} \xi \sin (e)\right] \phi_{0}=0 \\
& {\left[D_{1}(e)-l_{1}\right] R_{1}-\left[\left(B_{2}+B_{3}\right) \sin (e) \cos (e)-2 \mathrm{i} c_{1} \frac{\Omega}{k_{1}} \rho\right] P_{1}} \\
& -\mathrm{i}\left[\frac{\mathrm{i}}{k_{1}} B_{1}^{*} \cos (e)\right] \phi_{0}^{*}-\left[\frac{\mathrm{i}}{k_{1}} \xi \sin (e)\right] \phi_{0}=0 \\
& {\left[D_{1}(f)-l_{2}\right] R_{2}-\left[\left(B_{2}+B_{3}\right) \sin (f) \cos (f)-2 \mathrm{i} c_{2} \frac{\Omega}{k_{2}} \rho\right] P_{2}} \\
& -\mathrm{i}\left[\frac{\mathrm{i}}{k_{2}} B_{1}^{*} \cos (f)\right] \phi_{0}^{*}-\left[\frac{\mathrm{i}}{k_{2}} \xi \sin (f)\right] \phi_{0}=0 \\
& {\left[D_{1}(g)-l_{3}\right] R_{3}-\left[\left(B_{2}+B_{3}\right) \sin (g) \cos (g)-2 \mathrm{i} c_{3} \frac{\Omega}{k_{3}} \rho\right] P_{3}} \\
& -\mathrm{i}\left[\frac{\mathrm{i}}{k_{3}} B_{1}^{*} \cos (g)\right] \phi_{0}^{*}-\left[\frac{\mathrm{i}}{k_{3}} \xi \sin (g)\right] \phi_{0}=0 \\
& {\left[D_{1}(h)-l_{4}\right] R_{4}-\left[\left(B_{2}+B_{3}\right) \sin (h) \cos (h)-2 \mathrm{i} c_{4} \frac{\Omega}{k_{4}} \rho\right] P_{4}} \\
& -\mathrm{i}\left[\frac{\mathrm{i}}{k_{4}} B_{1}^{*} \cos (h)\right] \phi_{0}^{*}-\left[\frac{\mathrm{i}}{k_{4}} \xi \sin (h)\right] \phi_{0}=0
\end{aligned}
$$

Equation (4.2) is made possible using Snell's law such that: $c_{1} k_{0}=c_{1} k_{1}=c_{2} k_{2}=c_{3} k_{3}=c_{4} k_{4}$ and also $k_{0} \sin (e)=k_{1} \sin (e)=k_{2} \sin (f)=k_{3} \sin (g)=k_{4} \sin (h)$. Observe that $k_{0}=k_{1}$ and $c_{i}$, $i=1,2,3,4$, are functions of material parameters. We can rewrite Eq. (4.2) as follows 


$$
\begin{array}{lr}
R_{0}=-b_{1} P_{0}+b_{2} \phi_{0}^{*}+b_{3} \phi_{0} & R_{1}=b_{1} P_{1}+b_{4} \phi_{0}^{*}+b_{5} \phi_{0} \\
R_{2}=b_{6} P_{2}+b_{7} \phi_{0}^{*}+b_{8} \phi_{0} & R_{3}=b_{9} P_{3}+b_{10} \phi_{0}^{*}+b_{11} \phi_{0} \\
R_{4}=b_{12} P_{4}+b_{13} \phi_{0}^{*}+b_{14} \phi_{0} &
\end{array}
$$

where

$$
\begin{aligned}
& b_{1}=\frac{\left(B_{2}+B_{3}\right) \sin (e) \cos (e)+\left(2 \mathrm{i} c_{1} \Omega \rho\right) / k_{1}}{D_{1}(e)-l_{1}} \quad b_{2}=\frac{\mathrm{i} B_{1}^{*} \cos (e)}{k_{0}\left[D_{1}(e)-l_{1}\right]} \\
& b_{3}=\frac{\mathrm{i} \xi \sin (e)}{k_{0}\left[D_{1}(e)-l_{1}\right]} \quad b_{4}=\frac{\mathrm{i} \xi B_{1}^{*} \cos (e)}{k_{1}\left[D_{1}(e)-l_{1}\right]} \quad b_{5}=\frac{\mathrm{i} \xi \sin (e)}{k_{1}\left[D_{1}(e)-l_{1}\right]} \\
& b_{6}=\frac{\left(B_{2}+B_{3}\right) \sin (f) \cos (f)-\left(2 \mathrm{i} c_{2} \Omega \rho\right) / k_{2}}{\left.D_{1}(f)-l_{2}\right)} \quad b_{7}=\frac{\mathrm{i} B_{1}^{*} \cos (f)}{k_{2}\left[D_{1}(f)-l_{2}\right]} \\
& b_{8}=\frac{\mathrm{i} \xi \sin (f)}{k_{2}\left[D_{1}(f)-l_{2}\right]} \quad b_{9}=\frac{\left(B_{2}+B_{3}\right) \sin (g) \cos (g)-\left(2 \mathrm{i} c_{3} \Omega \rho\right) / k_{3}}{D_{1}(g)-l_{3}} \\
& b_{10}=\frac{\mathrm{i} B_{1}^{*} \cos (g)}{k_{3}\left[D_{1}(g)-l_{3}\right]} \quad b_{11}=\frac{\mathrm{i} \xi \sin (g)}{k_{3}\left[D_{1}(g)-l_{3}\right]} \\
& b_{12}=\frac{\left(B_{2}+B_{3}\right) \sin (h) \cos (h)-\left(2 \mathrm{i} c_{4} \Omega \rho\right) / k_{4}}{D_{1}(h)-l_{4}} \quad b_{13}=\frac{\mathrm{i} B_{1}^{*} \cos (h)}{k_{4}\left[D_{1}(h)-l_{4}\right]} \\
& b_{14}=\frac{\mathrm{i} \xi \sin (h)}{k_{4}\left[D_{1}(h)-l_{4}\right]} \quad l_{1}=\frac{\Omega^{2}}{k_{1}^{2}} \rho+c_{1}^{2}\left(\varepsilon_{0} \mu_{0}^{2} H_{0}^{2}+\rho\right) \\
& l_{2}=\frac{\Omega^{2}}{k_{2}^{2}} \rho+c_{2}^{2}\left(\varepsilon_{0} \mu_{0}^{2} H_{0}^{2}+\rho\right) \quad l_{3}=\frac{\Omega^{2}}{k_{3}^{2}} \rho+c_{3}^{2}\left(\varepsilon_{0} \mu_{0}^{2} H_{0}^{2}+\rho\right) \\
& l_{4}=\frac{\Omega^{2}}{k_{4}^{2}} \rho+c_{4}^{2}\left(\varepsilon_{0} \mu_{0}^{2} H_{0}^{2}+\rho\right) \quad
\end{aligned}
$$

\subsection{Free boundary conditions at the surface of the medium}

We consider a free surface boundary at $x_{2}=0$ of the micropolar fibre-reinforced rotating semi-infinite medium permeated by a uniform magnetic field with voids, i.e.: $\sigma_{i 2}+\bar{\sigma}_{i 2}=0$, $i=1,2, m_{23}=0$, and $\phi_{, 2}=0$ at $x_{2}=0$. Maxwell's stresses (Abd-Alla et al., 2017) are

$$
\begin{aligned}
& \bar{\sigma}_{i j}=\mu_{0} H_{0}\left(H_{i} h_{j}+H_{j} h_{i}-H_{k} h_{k} \delta_{i j}\right) \quad \Rightarrow \quad \bar{\sigma}_{i j}=\mu_{0} H_{0}\left|\begin{array}{ccc}
-h_{3} & 0 & h_{1} \\
0 & -h_{3} & h_{2} \\
h_{1} & h_{2} & h_{3}
\end{array}\right| \\
& h_{3}=-H_{0}\left(u_{1,1}+u_{2,2}\right) h_{1}=h_{2}=0
\end{aligned}
$$

and

$$
\begin{aligned}
& \text { for } i=1: \quad \sigma_{12}+\bar{\sigma}_{12}=0 \quad \text { but } \quad \bar{\sigma}_{12}=0 \Rightarrow \sigma_{12}=0 \\
& \text { for } i=2: \quad \sigma_{22}+\bar{\sigma}_{22}=0 \Rightarrow \sigma_{22}+\mu_{0} H_{0}^{2}\left(u_{1,1}+u_{2,2}\right)=0 \\
& m_{23}=0 \Rightarrow \phi_{3,2}^{*}=0 \quad \phi_{, 2}=0
\end{aligned}
$$

Thus, from equation (4.3), we obtain the ratio

$$
\begin{array}{ll}
\frac{R_{1}}{R_{0}}=\frac{1}{\varsigma}\left(\frac{b_{1} P_{1}}{P_{0}}+\frac{b_{4} \phi_{0}^{*}}{P_{0}}+\frac{b_{5} \phi_{0}}{P_{0}}\right) & \frac{R_{2}}{R_{0}}=\frac{1}{\varsigma}\left(\frac{b_{6} P_{2}}{P_{0}}+\frac{b_{7} \phi_{0}^{*}}{P_{0}}+\frac{b_{8} \phi_{0}}{P_{0}}\right) \\
\frac{R_{3}}{R_{0}}=\frac{1}{\varsigma}\left(\frac{b_{9} P_{3}}{P_{0}}+\frac{b_{10} \phi_{0}^{*}}{P_{0}}+\frac{b_{11} \phi_{0}}{P_{0}}\right) & \frac{R_{4}}{R_{0}}=\frac{1}{\varsigma}\left(\frac{b_{12} P_{4}}{P_{0}}+\frac{b_{13} \phi_{0}^{*}}{P_{0}}+\frac{b_{14} \phi_{0}}{P_{0}}\right) \\
\varsigma=\frac{b_{2} \phi_{0}^{*}}{P_{0}}+\frac{b_{3} \phi_{0}}{P_{0}}-b_{1} &
\end{array}
$$


and also by using Eqs. (4.4), we have

$$
\begin{aligned}
& \left(a_{11} P_{1}+a_{12} P_{2}+a_{13} P_{3}+a_{14} P_{4}\right) \frac{1}{P_{0}}=a_{15} \\
& \left(a_{21} P_{1}+a_{22} P_{2}-a_{23} P_{3}+a_{24} P_{4}\right) \frac{1}{P_{0}}=-a_{25} \\
& \left(a_{31} P_{1}-a_{32} P_{2}+a_{33} P_{3}+a_{34} P_{4}\right) \frac{1}{P_{0}}=-a_{35} \\
& \left(a_{41} P_{1}+a_{42} P_{2}+a_{43} P_{3}-a_{44} P_{4}\right) \frac{1}{P_{0}}=a_{45}
\end{aligned}
$$

where

$$
\begin{aligned}
& a_{11}=k_{1} \sin (e) \quad a_{12}=k_{2} \sin (f) \quad a_{13}=k_{3} \sin (g) \quad a_{14}=k_{4} \sin (h) \\
& a_{15}=-k_{0} \sin (e)-\frac{\mathrm{i} \phi_{0}^{*}}{P_{0}} \quad a_{21}=\left[L_{1} b_{1} \sin (e)-L_{2} \cos (e)\right] k_{1} \\
& a_{22}=\left[L_{1} b_{6} \sin (f)-L_{2} \cos (f)\right] k_{2} \quad a_{23}=-\left[L_{1} b_{9} \sin (g)-L_{2} \cos (g)\right] k_{3} \\
& a_{24}=\left[L_{1} b_{12} \sin (h)-L_{2} \cos (h)\right] k_{4} \\
& a_{25}=-\left[L_{1} b_{1} \sin (e)-L_{2} \cos (e)\right] k_{0}-L_{1}\left\{\left(b_{2} \frac{\phi_{0}^{*}}{P_{0}}+b_{3} \frac{\phi_{0}}{P_{0}}\right) k_{0} \sin (e)\right. \\
& +\left(b_{4} \frac{\phi_{0}^{*}}{P_{0}}+b_{5} \frac{\phi_{0}}{P_{0}}\right) k_{1} \sin (e)+\left(b_{7} \frac{\phi_{0}^{*}}{P_{0}}+b_{8} \frac{\phi_{0}}{P_{0}}\right) k_{2} \sin (f)+\left(b_{10} \frac{\phi_{0}^{*}}{P_{0}}+b_{11} \frac{\phi_{0}}{P_{0}}\right) k_{3} \sin (g) \\
& \left.+\left(b_{13} \frac{\phi_{0}^{*}}{P_{0}}+b_{14} \frac{\phi_{0}}{P_{0}}\right) k_{4} \sin (h)\right\}+\mathrm{i} \xi \frac{\phi_{0}}{P_{0}} \\
& a_{31}=-B_{1} b_{1} a_{11}^{2}+\left(B_{2}+B_{3}\right) a_{11} k_{1} \cos (e)+2 \mathrm{i} \rho c_{1} k_{1} \Omega-B_{4} b_{1} k_{1}^{2} \cos ^{2}(e) \\
& +\left[\Omega^{2} \rho+c_{1}^{2} k_{1}^{2}\left(\varepsilon_{0} \mu_{0}^{2} H_{0}^{2}+\rho\right)\right] b_{1} \\
& a_{32}=-\left\{-B_{1} b_{6} a_{12}^{2}+\left(B_{2}+B_{3}\right) a_{12} k_{2} \cos (f)+2 \mathrm{i} \rho c_{2} k_{2} \Omega-B_{4} b_{6} k_{2}^{2} \cos ^{2}(f)\right. \\
& \left.+\left[\Omega^{2} \rho+c_{2}^{2} k_{2}^{2}\left(\varepsilon_{0} \mu_{0}^{2} H_{0}^{2}+\rho\right)\right] b_{6}\right\} \\
& a_{33}=-B_{1} b_{9} a_{13}^{2}+\left(B_{2}+B_{3}\right) a_{13} k_{3} \cos (g)+2 \mathrm{i} \rho c_{3} k_{3} \Omega-B_{4} b_{9} k_{3}^{2} \cos ^{2}(g) \\
& +\left[\Omega^{2} \rho+c_{3}^{2} k_{3}^{2}\left(\varepsilon_{0} \mu_{0}^{2} H_{0}^{2}+\rho\right)\right] b_{9} \\
& a_{34}=-B_{1} b_{12} a_{14}^{2}+\left(B_{2}+B_{3}\right) a_{14} k_{4} \cos (h)+2 \mathrm{i} \rho c_{4} k_{4} \Omega-B_{4} b_{12} k_{1}^{2} \cos ^{2}(h) \\
& +\left[\Omega^{2} \rho+c_{4}^{2} k_{4}^{2}\left(\varepsilon_{0} \mu_{0}^{2} H_{0}^{2}+\rho\right)\right] b_{12} \\
& a_{35}=-\left\{B _ { 1 } \left[\left(-b_{1}+b_{2} \frac{\phi_{0}^{*}}{P_{0}}+b_{3} \frac{\phi_{0}}{P_{0}}\right) k_{0}^{2} \sin ^{2}(e)+\left(b_{4} \frac{\phi_{0}^{*}}{P_{0}}+b_{5} \frac{\phi_{0}}{P_{0}}\right) a_{11}^{2}+\left(b_{7} \frac{\phi_{0}^{*}}{P_{0}}+b_{8} \frac{\phi_{0}}{P_{0}}\right) a_{12}^{2}\right.\right. \\
& \left.+\left(b_{10} \frac{\phi_{0}^{*}}{P_{0}}+b_{11} \frac{\phi_{0}}{P_{0}}\right) a_{13}^{2}+\left(b_{13} \frac{\phi_{0}^{*}}{P_{0}}+b_{14} \frac{\phi_{0}}{P_{0}}\right) a_{14}^{2}\right]+\left(B_{2}+B_{3}\right) k_{0}^{2} \cos ^{2}(e)+2 \mathrm{i} \rho c_{1} \Omega \\
& +B_{4}\left[\left(-b_{1}+b_{2} \frac{\phi_{0}^{*}}{P_{0}}+b_{3} \frac{\phi_{0}}{P_{0}}\right) k_{0}^{2} \cos ^{2}(e)+\left(b_{4} \frac{\phi_{0}^{*}}{P_{0}}+b_{5} \frac{\phi_{0}}{P_{0}}\right) k_{1}^{2} \cos ^{2}(e)\right. \\
& +\left(b_{7} \frac{\phi_{0}^{*}}{P_{0}}+b_{8} \frac{\phi_{0}}{P_{0}}\right) k_{2}^{2} \cos ^{2}(f)+\left(b_{10} \frac{\phi_{0}^{*}}{P_{0}}+b_{11} \frac{\phi_{0}}{P_{0}}\right) k_{3}^{2} \cos ^{2}(g) \\
& \left.+\left(b_{13} \frac{\phi_{0}^{*}}{P_{0}}+b_{14} \frac{\phi_{0}}{P_{0}}\right) k_{4}^{2} \cos ^{2}(h)\right]-\left(-b_{1}+b_{2} \frac{\phi_{0}^{*}}{P_{0}}+b_{3} \frac{\phi_{0}}{P_{0}}\right)\left[\Omega^{2} \rho+c_{1}^{2} k_{0}^{2}\left(\varepsilon_{0} \mu_{0}^{2} H_{0}^{2}+\rho\right)\right] \\
& -\left(b_{4} \frac{\phi_{0}^{*}}{P_{0}}+b_{5} \frac{\phi_{0}}{P_{0}}\right)\left[\Omega^{2} \rho+c_{1}^{2} k_{1}^{2}\left(\varepsilon_{0} \mu_{0}^{2} H_{0}^{2}+\rho\right)\right]-\left(b_{7} \frac{\phi_{0}^{*}}{P_{0}}+b_{8} \frac{\phi_{0}}{P_{0}}\right)\left[\Omega^{2} \rho+c_{2}^{2} k_{2}^{2}\left(\varepsilon_{0} \mu_{0}^{2} H_{0}^{2}+\rho\right)\right] \\
& -\rho\left(b_{10} \frac{\phi_{0}^{*}}{P_{0}}+b_{11} \frac{\phi_{0}}{P_{0}}\right)\left[\Omega^{2} \rho+c_{3}^{2} k_{3}^{2}\left(\varepsilon_{0} \mu_{0}^{2} H_{0}^{2}+\rho\right)\right] \\
& -\rho\left(b_{13} \frac{\phi_{0}^{*}}{P_{0}}+b_{14} \frac{\phi_{0}}{P_{0}}\right)\left[\Omega^{2} \rho+c_{4}^{2} k_{4}^{2}\left(\varepsilon_{0} \mu_{0}^{2} H_{0}^{2}+\rho\right)\right]-\mathrm{i} \xi \frac{\phi_{0}}{P_{0}}\left[k_{0} \sin (e)+a_{11}+a_{12}+a_{13}+a_{14}\right]
\end{aligned}
$$




$$
\begin{aligned}
a_{41} & =-\left(B_{5}+B_{6}\right) a_{11}^{2}+\left[B_{2} a_{11} k_{1} \cos (e)-2 \mathrm{i} \rho c_{1} k_{1} \Omega\right] b_{1}+\Omega^{2} \rho+c_{1}^{2} k_{1}^{2}\left(\varepsilon_{0} \mu_{0}^{2} H_{0}^{2}+\rho\right) \\
a_{42} & =-\left(B_{5}+B_{6}\right) a_{12}^{2}+\left[B_{2} a_{12} k_{2} \cos (f)-2 \mathrm{i} \rho c_{2} k_{2} \Omega\right] b_{6}+\Omega^{2} \rho+c_{2}^{2} k_{2}^{2}\left(\varepsilon_{0} \mu_{0}^{2} H_{0}^{2}+\rho\right) \\
a_{43} & =-\left(B_{5}+B_{6}\right) a_{13}^{2}+\left[B_{2} a_{13} k_{3} \cos (g)-2 \mathrm{i} \rho c_{2} k_{3} \Omega\right] b_{9}+\Omega^{2} \rho+c_{3}^{2} k_{3}^{2}\left(\varepsilon_{0} \mu_{0}^{2} H_{0}^{2}+\rho\right) \\
a_{44} & =-\left\{-\left(B_{5}+B_{6}\right) a_{14}^{2}+\left[B_{2} a_{14} k_{4} \cos (h)-2 \mathrm{i} \rho c_{2} k_{4} \Omega\right] b_{12}+\Omega^{2} \rho+c_{4}^{2} k_{4}^{2}\left(\varepsilon_{0} \mu_{0}^{2} H_{0}^{2}+\rho\right)\right\} \\
a_{45} & =\left[B_{5} \sin ^{2}(e)+B_{6} \cos ^{2}(e)\right] k_{0}^{2}-\Omega^{2} \rho+c_{1}^{2} k_{0}^{2}\left(\varepsilon_{0} \mu_{0}^{2} H_{0}^{2}+\rho\right) \\
& +\left(b_{2} \frac{\phi_{0}^{*}}{P_{0}}+b_{3} \frac{\phi_{0}}{P_{0}}\right)\left[B_{2} k_{0}^{2} \cos (e) \sin (e)-2 \mathrm{i} \rho c_{1} k_{1} \Omega\right] \\
& -\left(b_{4} \frac{\phi_{0}^{*}}{P_{0}}+b_{5} \frac{\phi_{0}}{P_{0}}\right)\left[k_{1} B_{2} a_{11} \cos (e)-2 \mathrm{i} \rho c_{1} k_{1} \Omega\right] \\
& -\left(b_{7} \frac{\phi_{0}^{*}}{P_{0}}+b_{8} \frac{\phi_{0}}{P_{0}}\right)\left[k_{2} B_{2} a_{12} \cos (f)-2 \mathrm{i} \rho c_{2} k_{2} \Omega\right] \\
& -\left(b_{10} \frac{\phi_{0}^{*}}{P_{0}}+b_{11} \frac{\phi_{0}}{P_{0}}\right)\left[B_{2} k_{3} a_{13} \cos (g)-2 \mathrm{i} \rho c_{3} k_{3} \Omega\right] \\
& -\left(b_{13} \frac{\phi_{0}^{*}}{P_{0}}+b_{14} \frac{\phi_{0}}{P_{0}}\right)\left[k_{4} B_{2} a_{15} \cos (h)-2 \mathrm{i} \rho c_{4} k_{4} \Omega\right] \\
& +\mathrm{i} B_{5} \frac{\phi_{0}^{*}}{P_{0}}\left[k_{0} \sin (e)+a_{11}+a_{12}+a_{13}+a_{14}\right] \\
L_{1} & =B_{2} \quad L_{2}=B_{6}
\end{aligned}
$$

Equation (4.6) can be solved for $P_{i} / P_{0}, i=1,2,3,4$ using Crammer's rule or any solver. $W_{i}=\left|P_{i} / P_{0}\right|, i=1,2,3,4$, give the amplitude ratios along the vertical reflection component. Hence, the amplitude ratios $Z_{i}=\left|R_{i} / R_{0}\right|, i=1,2,3,4$, along the horizontal reflection component can be obtained from Eqs. (4.5) by substituting the values of $P_{i} / P_{0}, i=1,2,3,4$.

\section{Computational results and discussion}

The effects of rotation, reinforcement and voids parameters on reflection coefficients of plane waves in the micropolar fibre-reinforced material under the influence of a magnetic field are studied by considering the following numerical physical constants (Othman and Lotfy, 2013) and other parameters as: $\lambda=7.59 \cdot 10^{9} \mathrm{~N} / \mathrm{m}^{2}, \mu_{T}=1.89 \cdot 10^{9} \mathrm{~N} / \mathrm{m}^{2}, \mu_{L}=2.45 \cdot 10^{9} \mathrm{~N} / \mathrm{m}^{2}$, $\rho=7800 \mathrm{Kg} / \mathrm{m}^{3}, \alpha=-1.28 \cdot 10^{9} \mathrm{~N} / \mathrm{m}^{2}, \beta=0.32 \cdot 10^{9} \mathrm{~N} / \mathrm{m}^{2}, \xi=220.90 \cdot 10^{9} \mathrm{~N} / \mathrm{m}^{2}$, $H_{0}=100 \mathrm{~A} / \mathrm{m}, J k^{2}=1, \Omega=50 / \mathrm{s}$.

Using Snell's law, the angle of reflections and the wave numbers are calculated. Also the speeds of waves; $c_{1}, c_{2}, c_{3}$ and $c_{4}$ for qID, qTD, qTM and the wave due to voids, respectively, are obtained from quartic Eq. (3.3). Thus, the graphs are presented in Figs. 2-4. That is, we considered variations of rotation $\Omega$ (angular velocity) of the medium, magnetic field, and voids $\xi$ parameters. This is to ascertain the effects of these parameters on the horizontal reflection coefficients $\left(\mathrm{RC}\right.$ or $\left.Z_{i}\right)$ of the reflected waves when, in particular, the $\mathrm{P}$-wave is incident with angle $e$.

Figures $2 \mathrm{a}-2 \mathrm{~d}$ represents the effects of rotation to the reflection coefficients $Z_{i}, i=1,2,3,4$ of the waves (qlD, qTD, qTM and due to voids) under constant magnetic fields and voids parameters. Also it suffices that the reflection coefficients have its maximum close to the normal incidence angle of the wave in the medium. Consequently, Figs. 2a-2d depict that the reflection coefficients increase when the rotation parameter increases. Though, the reflection coefficients in Figs. 2a-2d show very slight uniform behavior near $e>40^{\circ}$ as it vanishes at grazing angles of incidence. Moreover, an increase in the angle of incidence, decreases the reflection coefficients in all cases due to reinforcement of the medium. However, it entails that the propagation of waves at different points in the medium with an increase in rotation will decrease the speed of waves 
except for the wave due to voids (Fig. 2f), which is unaffected at all points of propagation. Also, as the angle of propagation increases, the speed of waves increases. The speed of waves vanishes at the grazing angle of incidence, which is the same for the coefficients of reflected waves. Hence, the dependencies of the constituent parameters of the system are proportional.

(a)

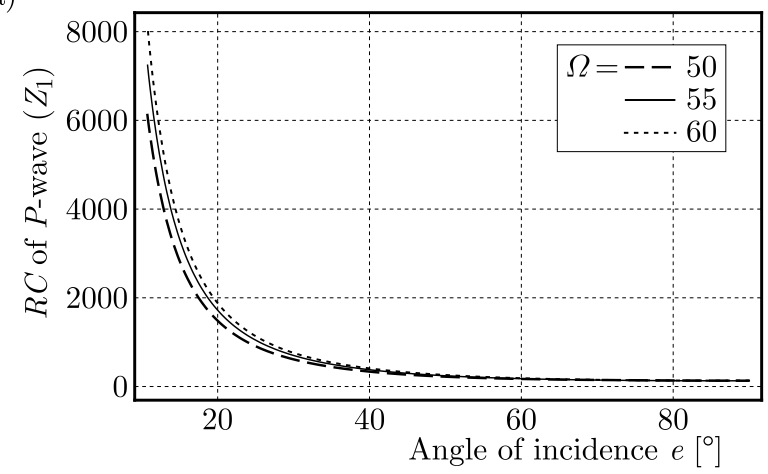

(c)

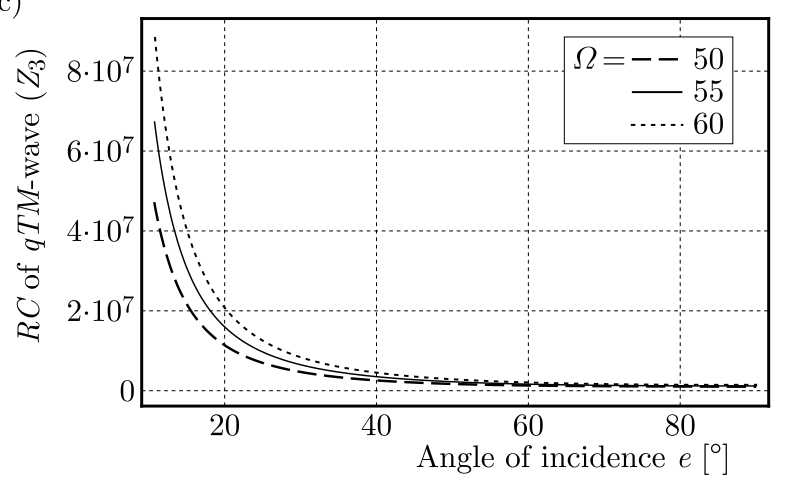

(e)

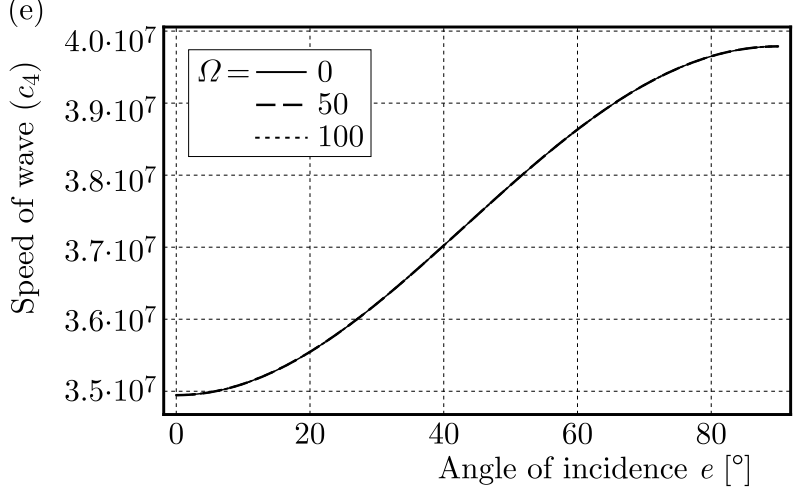

(b)

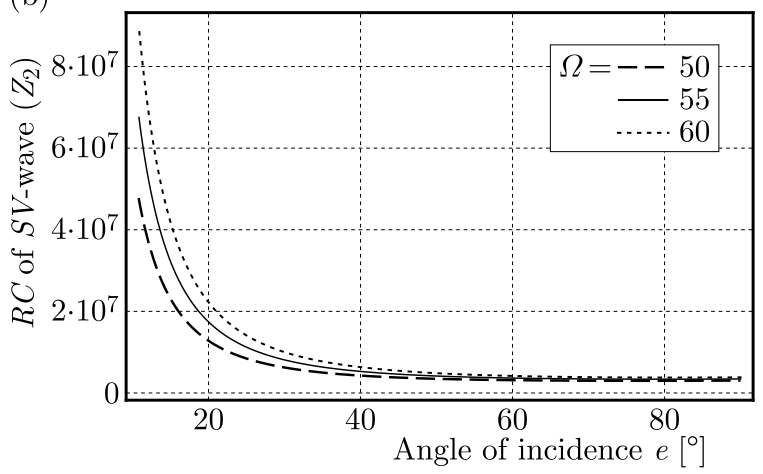

(d)

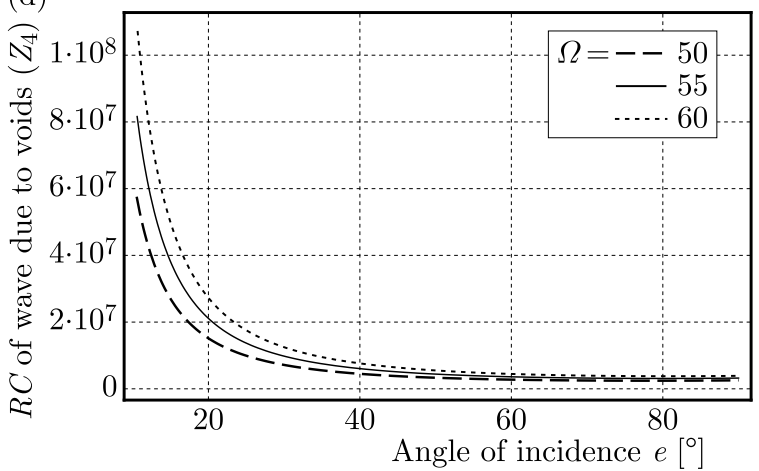

Fig. 2. Variations of the reflection coefficients $Z_{i}$ and speed of waves versus the incidence angle for distinct values of rotation $\Omega$

It is observed in Figs. 3a-3d that the reflection coefficients $Z_{i}(R C), i=1,2,3,4$ of qLD (P-wave), qTD (SV-wave), qTM (quasi-transverse microrotational wave) and wave due to voids, respectively, decrease for the increasing angle of incidence for uniform rotation and voids parameters. They have sharp and fast increasing behavior in the presence of constant rotation and voids effects with respect to varying high magnetic field $H_{0}$ effects, except for $Z_{3}$ which decreases. In Fig. $3 Z_{i}(R C), i=1,2,3,4$ start with their curves at maximum values close to the normal incidence and, afterwards, decrease slightly when the angle of incidence varies near $e>45^{\circ}$ and, finally, vanish at grazing incidence. Thus, at some points in the medium, varying magnetic field effects could lead to a decrease or increase in the reflection coefficients of the waves with combined uniform porosity and rotation of the medium. Furthermore, for higher 
magnetic field, reflection of the wave is possible and its reflection coefficients may possess small or high values in such a medium. Thus, for an increased incidence angle, reflection coefficients in the mircopolar fibre-reinforced medium yield low values in the presence of rotation and voids.

(a)

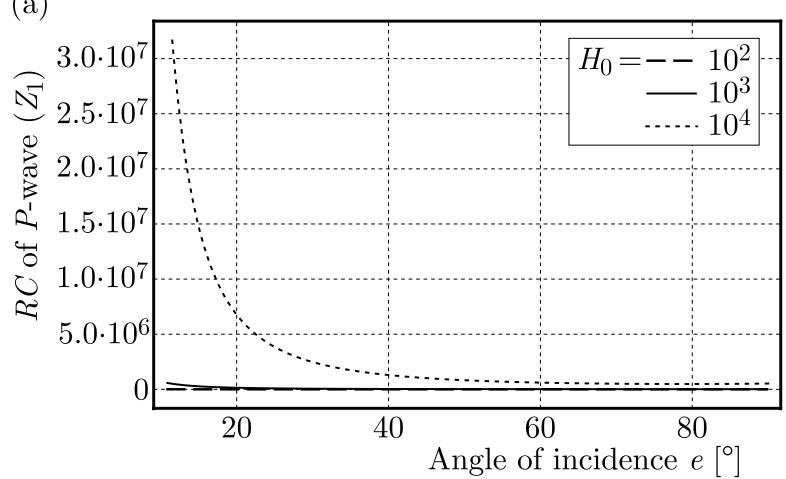

(c)

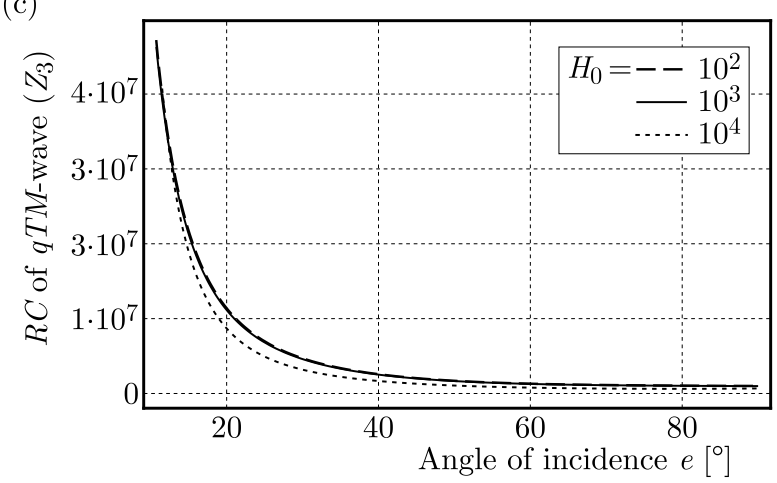

(b)

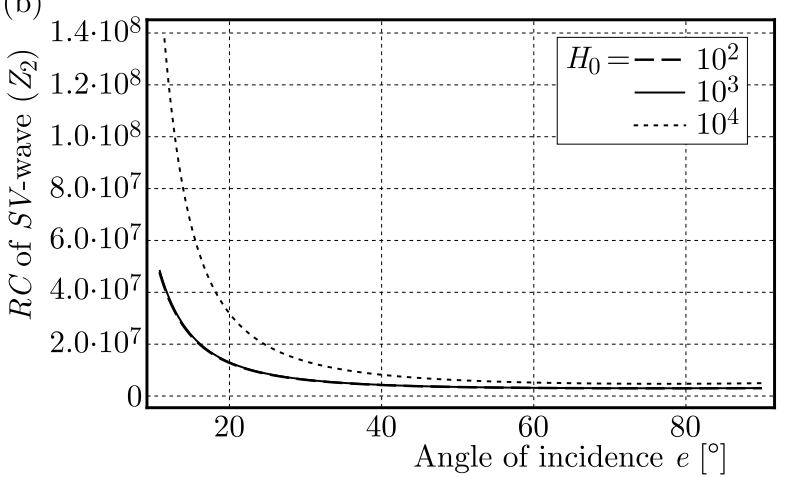

(d)

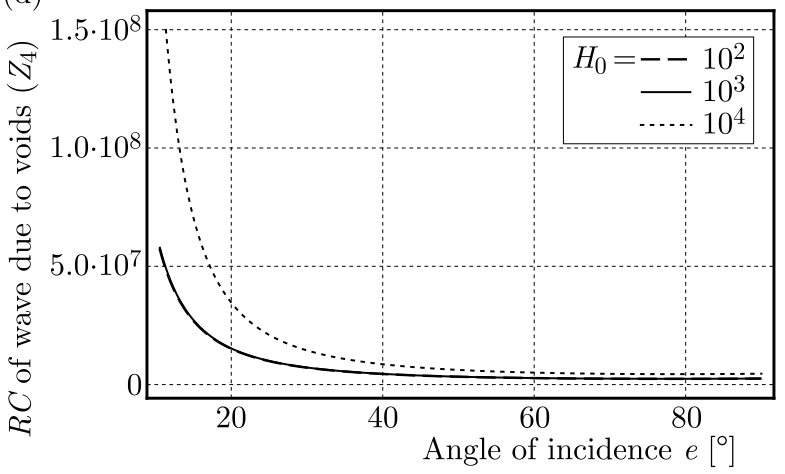

Fig. 3. Variations of the reflection coefficients $Z_{i}$ versus the incidence angle for distinct values of $H_{0}$

(a)

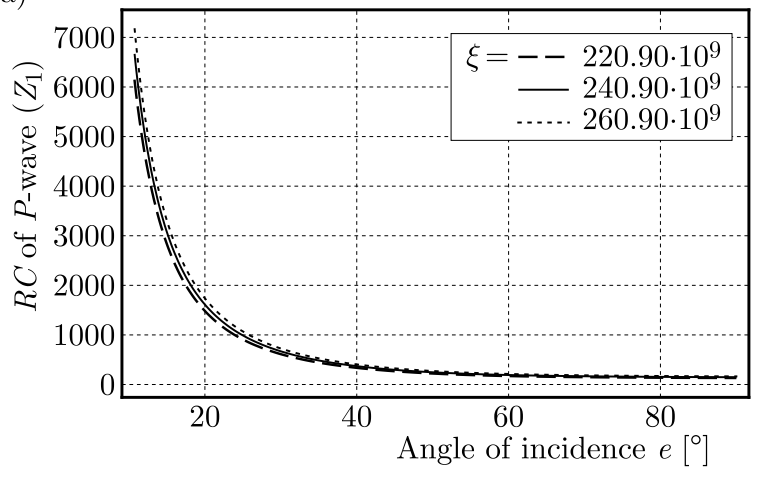

(c)

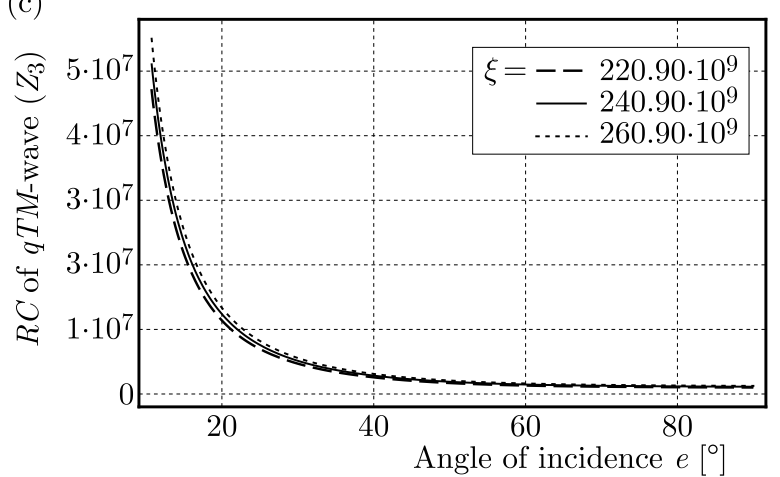

(b)

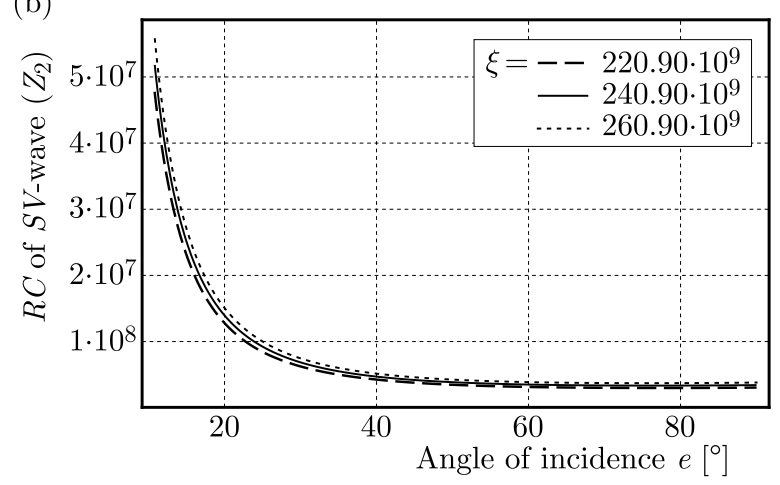

(d)

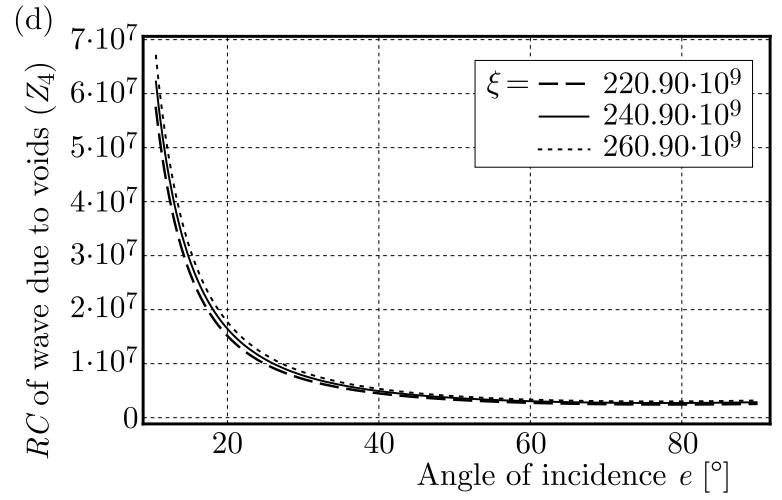

Fig. 4. Variations of the reflection coefficients $Z_{i}$ versus the incidence angle for distinct values of $\xi$ parameter 
Furthermore, Figs. 4a-4d depict variation of reflection coefficients $Z_{i}(R C), i=1,2,3,4$ of qLD (P-wave), qTD (SV-wave), qTM (quasi-transverse microrotational wave) and the wave due to voids, respectively, for uniform rotation and magnetic field parameters. They are increasing with respect to an increased voids parameter $\xi$ in the presence of a constant applied magnetic field and rotation. They decrease when the angle of incidence increases due to the mircopolar fibre-reinforced medium. Figure 4 shows the curves at maximum values close to the normal incidence and, subsequently, decreasing very slightly and finally vanishing at grazing incidence.

\section{Conclusion}

We investigated the propagation and reflection of magneto-elastic plane waves at free surfaces of a rotating micropolar fibre-reinforced medium with porosity. Four coupled reflected plane waves traveling in the medium; quasi-longitudinal displacement (qLD) wave, quasi-transverse displacement (qTD) wave, quasi-transverse microrotational (qTM) wave and a wave due to voids were observed. The characteristics of the propagation and reflection of the waves were made up of angular velocity of the medium $\Omega$, micropolar fibre-reinforced and voids parameters under the influence of magnetic fields. It was deduced that these parameters greatly influenced the reflection coefficients $Z_{i}$, speeds and modulation of waves. It means that the micropolar fibrereinforcement tends to decrease the reflection coefficients in the medium with effects of rotation, magnetic fields and voids as the angle of incidence increases in the medium. The speeds of waves were affected by the rotation parameter by decreasing its propagation. Generally, the reflection coefficients $Z_{i}$, decreased for an increased incident angle due to the micropolar fibrereinforcement. It is noted that rotation increased at certain values the reflection coefficients while high magnetic field decreased the reflection coefficients $Z_{i}$ of reflected qTM waves in the modelled problem. Moreover, voids increasrd the reflection coefficients of $\mathrm{qLD}$, (P-wave), qTD (SV-wave) and qTM (quasi-transverse microrotational wave) and waves due to voids. Also it is deduced that reflection might not occur for certain incident angles in the medium. The method used in this study is noteworthy for dealing with such problems for successful investigation. Our modeled problem is similar to Sunita et al. (2019) if the magnetic field, micropolarity and voids are neglected in the presence of thermo-elasticity.

The investigation should prove to be helpful for new researchers in the field and experimental based study involving propagation and reflection of magneto-elastic plane waves at free surfaces of a rotating micropolar fibre-reinforced medium with voids and also in fields with similar models such as in optics, geophysics, design of new materials, etc.

\section{Acknowledgements}

The World Academy of Sciences (TWAS) and COMSATS University Islamabad are appreciated for supporting and making the research works a success (FR \#: 3240293223).

\section{References}

1. Abd-Alla A.M., Abo-Dahab S. M., Khan A., 2017, Rotational effects on magneto-thermoelastic Stoneley, Love, and Rayleigh waves in fibre-reinforced anisotropic general viscoelastic media of higher order, Computers, Materials and Continua, 53, 1, 49-72

2. Baljeet S., Sarva J.T., 2004, Reflection of plane waves at the free surface of a fibre-reinforced elastic half-space, Sādhan $\bar{a}, \mathbf{2 9}, 3,249-257$

3. Biswas P.K., Sengupta P.R., Debnath L., 1996, Axisymmetric Lamb's problem in a semi-infinite micropolar viscoelastic medium, International Journal of Mathematics and Mathematical Scieences, 19, 4, 815-820 
4. Chattopadhyay A., Choudhury S., 1990, Propagation, reflection and transmission of magneto elastic shear waves in a self-reinforced medium, International Journal of Engineering Science, 28, 6, 485-495

5. Chattopadhyay A., Venkateswarlu V.R.K., Saha S., 2002, Reflection of quasi-P and quasi-SV waves at the free and rigid boundaries of a fibre-reinforced medium, Sādhanā, 27, 6, 613-630

6. Chaudhary S., Kaushik V.P., Tomar S. K., 2004, Reflection/transmission of plane SH wave through a self-reinforced elastic layer between two half-spaces, Acta Geophysica Polonica, 52, 2, 219-235

7. Cowin S.C., Nunziato J.W., 1983, Linear elastic materials with voids, Journal of Elasticity, 13, 2, 125-147

8. ERINGEN A.C., 1967, Linear theory of micropolar viscoelasticity, International Journal of Engineering Science, 5, 2, 191-204

9. Hillion P., 2008, Harmonic plane wave propagation in anisotropic chiral media, International Journal of Applied Electromagnetics and Mechanics, 28, 3, 337-350

10. Khan A., Anya A.I., Kaneez H., 2015, Gravitational effects on surface waves in non-homogeneous rotating fibre-reinforced anisotropic elastic media with voids, International Journal of Applied Science and Engineering Research, 4, 5, 620-632

11. Kumar R., Gogna M.L., Debnath L., 1990, On Lamb's plane problem in a micropolar viscoelastic half-space with stretch, International Journal of Mathematics and Mathematical Scieences, 13, 2, 363-372

12. Kumar R., Sharma K.D., Garg S.K., 2015, Reflection of plane waves in transversely isotropic micropolar viscothermoelastic solid, Materials Physics and Mechanics, 22, 1, 1-14

13. LAkes R.S., Benedict R.L., 1982, Noncentrosymmetry in micropolar elasticity, International Journal of Engineering Sciences, 20, 10, 1161-1167

14. LATA P., 2018, Reflection and refraction of plane waves in a layered nonlocal elastic and anisotropic thermoelastic medium, Structural Engineering and Mechanics, 66, 1, 113-124

15. MCCArthy M.F., Eringen A.C., 1969, Micropolar viscoelastic waves, International Journal of Engineering Science, 7, 5, 447-458

16. Nunziato J.W., Cowin S.C., 1979, A nonlinear theory of elastic materials with voids, Archives Rational Mechanics and Analysis, 72, 2, 175-201

17. Oтнman M.I.A., Lotfy Kн., 2013, The effect of magnetic field and rotation of the 2-D problem of a fiber-reinforced thermoelastic under three theories with influence of gravity, Mechanics of Materials, 60, 129-143

18. Puri P., Cowin S.C., 1985, Plane waves in linear elastic materials with voids, Journal of Elasticity, 15, 2, 167-183

19. Roy I., ACharya D.P., Acharya S., 2017, Propagation and reflection of plane waves in a rotating magneto-elastic fibre-reinforced semi space with surface stress, Mechanics and Mechanical Engineering, 21, 4, 1043-1061

20. Schoenberg M., Censor D., 1973, Elastic waves in rotating media, Quarterly of Applied Mathematics, 31, 1, 115-125

21. Sengupta P.R., NAth S., 2001, Surface waves in fibre-reinforced anisotropic elastic media, Sādhan $\bar{a}, \mathbf{2 6}, 4,363-370$

22. Singh B., 2000, Reflection and transmission of plane harmonic waves at the interface between a liquid and micropolar viscoelastic solid with stretch, Sādhana - Academy Proceedings in Engineering Sciences, 25, 6, 589-600

23. Singh B., 2017, Reflection of elastic waves from plane surface of a half-space with impedance boundary conditions, Geosciences Research, 2, 4, 242-253 
24. Singh B., Kumar R., 1998, Reflection and refraction coefficient of plane waves at an interface between micropolar elastic solid and viscoelastic solid, International Journal of Engineering Science, 36, 2, 119-135

25. Sinha M., Bera R.K., 2003, Eigenvalue approach to study the effect of rotation and relaxation time in generalized thermoelasticity, Computers and Mathematics with Applications, 46, 783-792

26. Sunita D., Suresh K.S., Kapil K.K., 2019, Reflection at the free surface of fibre-reinforced thermoelastic rotating medium with two temperature and phase-lag, Applied Mathematics Modelling, 65, 106-119

27. TAuchert T.R., 1971, Thermal stresses in micropolar elastic solids, Acta Mechanica, 11, 3, 155-169

Manuscript received January 27, 2019; accepted for print May 6, 2019 